\title{
Psychological effects of boarding students in schools far away from home - a qualitative study of a sample of students referred to a psychiatry clinic from Central Sri Lanka
}

AS Malalagama

\section{Abstract \\ Background}

The education system in Sri Lanka drives parents to seek popular schools in cities for their children, some of who are being boarded far away from their homes (1). Living away from home at a young age can cause grief and even depression. Children boarded at an early age may present with a cluster of symptoms later in life known as "Boarding School Syndrome" (BSS) (2).

\section{Aims}

The aims of this study were to explore the psychological effects of being boarded in schools far away from home, in a sample of students referred to the psychiatry unit of Base Hospital Diyatalawa.

\section{Methods}

This was a qualitative study. Students who had been boarded in schools in four towns, who were referred to the psychiatry unit, and their parents, were interviewed using a semi-structured interview. Interviews were audio-recorded, transcribed and thematic analysis was done.

\section{Results}

Seven students were interviewed, aged between 1118 yrs with a mean age of 13.4 yrs. Several main themes were identified from the analysis; grief at leaving home, difficulty in transitioning to the new school environment, worry about effect on scholastic performance after boarding, crisis situations and cries for help, and the experience of emotional abuse. Several students also had features of depression.

\section{Conclusions}

The findings of this study suggest that separation from parents in childhood significantly increases the risk of negative psychological outcomes including depressive symptoms. This is in keeping with findings from similar previous studies. Psychiatrists should be aware of the possible negative impact of being separated from parents and boarded away from home, when assessing children and adolescents.

Key words: children and adolescents, boarding school syndrome, early separation, depression, deliberate self-harm

SL J Psychiatry 2020; 11(1): 14-19

\section{Introduction}

Most Sri Lankan (SL) parents aim to provide the best education they can afford, for their children. During the past decades, SL parents have been moving to cities in order to enrol their children in popular schools (1). When not in a position to move to a city, parents may choose to board their children either in boarding schools or in private boarding houses, in order to gain access to urban schools. Separation from parents and living away from home at a young age can cause grief and even depression in school children (2). Bullying or ragging, and discrimi- nation or punitive acts by teachers seen in some schools and boarding houses may further exacerbate this distress (2). Grief and depression are risk factors for self-harm in children and adolescents (3).

Children who have been boarded at an early age may present with psychological problems later in life. This cluster of symptoms, characterised by maladaptive defence mechanisms and difficulty in intimate relationships is known as "boarding school syndrome" (BSB) (2). The sudden and irrecoverable loss of primary attachments at an early age is considered to be the 
underlying cause $(2,4)$. When children are transitioning to boarding schools during the middle years, their social, emotional and academic wellbeing maybe adversely affected due to the changes they experience (5-8).

Among the many school children referred to the Psychiatry Unit of Base Hospital, Diyatalawa, there is an increasing trend of adolescents, who have been boarded for schooling, presenting with self-harm. There are several popular boarding schools in the towns close to Diyatalawa, including in Bandarawela, Nuwera Eliya and Welimada, from which students are referred for psychological problems. Most of these students live away from their homes and are visited by families and are not frequently visited by family members. It is important to study the psychological effects of being boarded in this group of students, in order to identify and minimize any psychological distress, which may lead to complications later in life. Available studies in this area are limited, both internationally and in Sri Lanka.

The aims of this study were to explore the psychological effects, including homesickness, depression, and deliberate self-harm (DSH), and the effect on scholastic performance, due to being boarded away from home, in a sample of students referred to the Psychiatry Unit of Base Hospital, Diyatalawa.

\section{Methods}

This was a qualitative study. Students boarded at schools in Diyatalawa, Bandarawela, Nuwera Eliya or Welimada, aged between 6-18 yrs, who were referred to the psychiatry unit of Base Hospital, Diyatalawa by other medical units, schools, law enforcement authorities or self, were considered eligible for inclusion in the study. A sample of 7 students who were referred consecutively to the study clinic, between November 2018 and March 2019 were interviewed. The study participants were all males, aged between 11-18 yrs.

Students were interviewed first by themselves, and then with their parents. The students and parents were interviewed using a semi structured interview guide, which was designed for the study. Areas of interest in the interview guide included, exploration of the any psychological difficulties experienced after being boarded, nature of relationship between the child and parents before and after being boarded, any bullying or ragging experienced, nature of relationship with school authorities and warden of the boarding house. All interviews were audiotaped and then transcribed.

Symptoms of depression and anxiety were assessed by clinical interview based on International Classification of Diseases (ICD-10) criteria. Risk of suicide was assessed using the interviewer administered Adolescent Suicide
Assessment Protocol (ASAP-20) which has been translated and validated for use among Sri Lankan adolescents (9).

Demographic details collected include gender, age, grade, hometown, distance to the boarding school from hometown, parents' occupations and education level, details of siblings, type of previous school and current school (according to the classification of state schools in Sri Lanka) and time period of being boarded in the current school (10).

Marks obtained and the position in the class during the last term test completed at the previous school, and at the current school, were recorded.

\section{Ethical aspects}

Ethical approval for the study was obtained from the Ethics Review Committee of the Faculty of Medicine, University of Peradeniya. Informed written consent was obtained from both students and parents of participants aged less than 18 years, and from the students aged $18 y r s$, prior to inclusion in the study.

All interviews were conducted in a confidential area. The identity of the students, parents and schools was not revealed at any time during the study and confidentiality of the students and parents were secured at all times. The data collected was accessible only to the principal investigator and the research assistant, and both written and recorded data was stored securely.

\section{Analysis}

Theoretical thematic analysis by Braun and Clarke was used as the guideline for analysis of the semi-structured interviews (11). The transcribed data were reviewed for recurring words, codes and themes. Each transcript was read through in entirety, and all segments of text that seemed relevant to the research question were coded. An inductive approach was used for coding. New codes were generated and codes were modified during the process. Codes were reviewed for patterns of themes manually, using the hard copies of transcripts.

The preliminary themes identified after sorting and collating the coded data extracts were, missing the family environment or home (homesickness), wanting to go back home, feelings of loneliness, dislike of the new school/ boarding house, not having friends in the new school, being ill treated by the teachers/warden, physical symptoms (feeling sick), not wanting to continue schooling, not being able to perform well at school, feeling sad, fearful/anxious, ideas to harm self/end life, help seeking behaviours, experience of bullying/ragging, anger towards parents after being boarded. These preliminary themes were reviewed with the help of a 
medical officer who did not participate in the interviews, to see whether they represented the coded data and the associated interview extracts. The themes were then refined and modified, collapsing some under a single theme. The initially identified themes of missing the family environment or home, wanting to go back home and feelings of loneliness, and anger towards parents after being boarded were combined under one theme which was defined as "Grief at leaving home". Dislike of the new school or boarding house, not having friends in the new school and not wanting to continue schooling were collated under the theme, "Difficulty in transitioning to the new school environment”. Concern about not being able to perform well at school was considered as a single theme, which was refined as "Worry on the effect on scholastic performance after boarding”. Ideas to harm self or end their life, and help seeking behaviours were combined to form the theme, "Crisis situations and cries for help”. Ill-treatment by teachers or wardens and the experience of bullying or ragging were considered as a separate theme, i.e., the "Experience of emotional abuse". Figure 1 shows the thematic map.

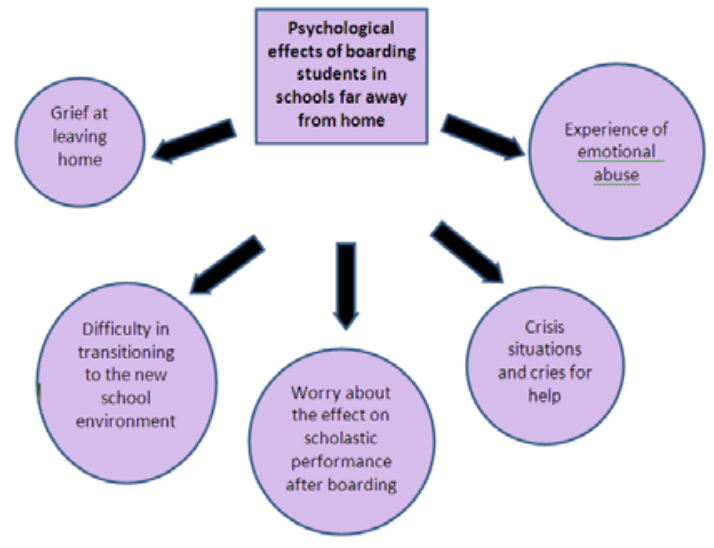

Figure 1. Map showing themes that emerged during the interviews.

\section{Results}

The seven students interviewed were aged between 11$18 y r$ with a mean age of 13.4 years. When considering the distance between the students' home and school, the farthest hometown was situated $204 \mathrm{~km}$ from the boarding school, while the nearest was $24 \mathrm{~km}$ away. On the date of the interview, the time duration spent at the new school ranged from one month to seven months, with the average being 3.6 months.

As described previously, interview analysis generated several major themes, which are described separately below.

\section{Grief at leaving home}

A prominent theme that emerged from all participants was that of missing home, parents, siblings, home environment, best friends and the previous school. The grief was expressed as anger, in a majority projected towards their parents. The following quote depicts the grief of one student.

"I often remember home. It was difficult without the parents being close to me. I remember how I played at home, and how I learned at my village school, which makes me sad. I think schooling at the village was a luxury. That environment is familiar to me, the teachers know me well. Sometimes I feel angry with my parents for bringing me here. I thought, why they are trying to keep me away from them?"

\section{Difficulty in transitioning to the new school environment}

Another prominent theme elicited was difficulty in adjusting to the new school environment. Most students expressed dislike of the new school, or hostel, or both. Difficulty in engaging with or building up relationships with peers in the new school and the hostel was the most common reason identified. They felt students in the new school were different from those at the previous school - in that they were less friendly, self-centred and less helpful - as exemplified by this student's comment:

"Students of the new school are not that friendly. They are not willing to lend their books. I felt they are much cleverer than me and that I am weak".

\section{Worry about the effect on scholastic performance after boarding}

Another theme that emerged among parents and students both was worry about lowering of the child's academic performance after being boarded. The most commonly attributed reasons for this, by students were, having to work in the English medium in the new school, faring less well compared to the other students in the new school, and staying away from home.

\section{Crisis situations and cries for help}

Four students presented after deliberate self-harm (DSH), by ingestion of paracetamol. The common theme was attempting self-harm as an attempt to get help or change their situation. One student described how he took the overdose as a cry for help, to make his parents take him back home:

"I thought that if I take an overdose of paracetamol, my parents would come to take me home. One of the students from this school took an overdose and was hospitalized. He was fine. Nothing happened to him. I didn't know that an overdose of paracetamol could kill you, but I knew that you will be hospitalized if you did so. I wanted to scare my parents, so that they know that I can't stay here”.

The students who engaged in DSH were from hometowns more than $100 \mathrm{~km}$ away from their new schools, and had been enrolled in the new school from one month to three months prior to the presentation. 
Two of the students who overdosed had not been visited by their parents since admission to the boarding house. These four students were found to have a moderate risk of suicide, with an ASAP-20 score of above 8.5 for each student (9).

\section{Experience of emotional abuse}

Although less commonly reported, another theme of importance was the experience of abuse. Three students revealed various forms of emotional abuse by teachers or peers in the new school - for example, negative comments on their origin in a rural area or about not being fluent in English. One student described it this way:

"One of the teachers in the school used to make bad comments whenever he sees me. He says that I am not behaving up to standards of the school. One day he asked me a question and when I couldn't answer, he scolded me in front of all the students, criticising me for coming from a rural school".

None of the students reported physical or sexual abuse.

Four out of seven students, who had also engaged in DSH, met ICD 10 criteria for mild or moderate depression (3). The onset of depressive symptoms followed enrolment in the boarding school. One student met criteria for social anxiety disorder (12).

\section{Discussion}

The findings of this study suggest that children and adolescents who are boarded away from home may undergo various deleterious psychological effects. Previous studies on psychological effects of early separation of children from their parents or primary attachment figures have mainly focused on grief following loss of loved ones or parental divorce (13). Evidence of effects of early separation following admission to boarding schools at an early age is more limited, with a few studies having been done internationally $(2,4,14)$. To the best of our knowledge, no Sri Lankan studies are available on this topic, to date. This study identified several important themes associated with the stressor of being boarded away from home.

\section{Grief at leaving home}

Grief at leaving home was an important theme that emerged from all the participants of this study. When children are sent away from home at an early age, they may suffer significant trauma due to sudden and often irrevocable loss of their primary attachments (14). In a home environment, families tend to adapt to the changes a child undergoes throughout development; but the reverse would happen in a boarding school, where the child has to adjust to a rigid system (14). The resultant psychological adverse effects have been reported to cause a cluster of long lasting symptoms and behaviours, which has been called the Boarding School Syndrome (BSS) by Joy Schaverien, a Jungian analyst and a psychotherapist (14). Later in life, the ex-boarder may experience a generalized sense of depression, broken relationships and marital or work related problems. This is a result of the distress originating in the losses and broken attachments of their early childhood (14).

\section{Difficulty in transitioning to the new school environment}

Experiencing an adjustment reaction during the initial stages of changing schools was observed in all participants and can be considered a normal reaction (15). However, the severity of the experience may be influenced by the resilience of the individual child as well as the nature of the change (16). Transition to a new school induces anxiety in students and can challenge their ability to cope with a new situation (17).

\section{Worry about the effect on scholastic performance after being boarded}

The decline in scholastic performance after being boarded at a new school could be a result of an adjustment disorder or depressive symptoms faced following the change. Students attributed their decline of performance to factors such as increased competitiveness and being away from home and were anxious about this. Anxiety itself may lower grades and decrease academic motivation when experiencing stresses of numerous changes (18).

\section{Crisis situations and cries for help}

DSH can be a form of cry for help among adolescents (18). Four of the participants appear to have taken overdoses as a cry for help - they describe their intent to get their parents' attention to the difficulties they were facing away from home. Failure to identify this distress experienced by boarded students, both by the school authorities and the parents, and the strict rules in the boarding houses that suppress expressions of distress, were contributing towards the distress emerging as DSH. These incidents of DSH are of significance as they posed a moderate suicide risk as assessed by the ASAP-20. The risk of repetition in the future in crisis situations is a high possibility (9). The depression identified in some students may increase this risk, and the early separation from parents may have been a risk factor for the depression noted in participants in this study (13).

\section{Experience of emotional abuse}

Another theme in this study was the experience of emotional abuse by children who were boarded. Emotional abuse by school teachers can cause significant negative consequences in the psychological and personality development of children (19). These forms of abuse may result in the child perceiving the new attachment figures around him to be unsafe (14). 
In Sri Lanka studying in a popular school with educational advantages is considered a privilege, even if it requires to be away from home. However, the findings of this study highlight that the perceived benefits of being boarded to attend a good school, need to be weighed against the long-term psychological adverse effects caused by damage to the primary attachments at an early age. International evidence suggests that while a majority of students who learn in these schools achieve significant social status in the long run due to educational advantages, some students may experience long lasting effects on their emotional, cognitive and behavioural patterns which may negatively affect their relationships, occupations, psychological well-being and be a risk for psychiatric disorders in the future (14).

Maldistribution of human and educational resources among rural and urban settings in Sri Lanka contributes towards the decision of parents to send their children to far away schools (1). Difficulty in accessing these urban areas from rural regions due to geographical characteristics and lack of effective transportation systems cause practical difficulties and may prevent the parents from visiting their children frequently. It is now timely for education and health authorities to collaborate and openly discuss this issue, in order to standardise the education system in Sri Lanka and provide equal opportunities for children islandwide, and thus minimize long-term negative psychological consequences in children due to separation from their families.

Furthermore, parents and educators need to be informed of strategies to help children cope with the grief of leaving home, and to inculcate methods of preparation to be away from home, such as involving children in the decision making, educating them on homesickness, establishing reliable modes of correspondence with home and encouraging to make new friends. Schools in the United States of America with extensive transition programs are shown to have significantly lower failure and dropout rates than those schools that did not offer comprehensive programs (20). The Sri Lankan school system should also be proactive and provide new students with orientation, counselling and peer support.

\section{Limitations}

This study was limited to seven participants, which was a relatively small sample and would have limited findings. However, in-depth interviews were conducted with each participant (and parents), which elicited detailed and significant information about this topic. Individual coping mechanisms, already existing psychological problems and temperament characteristics of the participants were not assessed in this study, which may have contributed to individual reactions and responses to separation from parents and home at an early age. Participants were those who had already been identified and referred for psychiatric support, which might have biased the sample. Further, the psychological impact of boarding may vary with the age of the child, which was not analysed. All participants were male, which was also a limitation - female students may have reacted differently. A separate analysis taking into account, the occupation level and education level of the parents was not performed. Furthermore, an analysis on whether parents' expected outcomes of changing schools were met, would have been useful.

\section{Conclusions}

The findings of this study supports results of previous studies which have shown that separation from parents in childhood, even for reasons other than parental death or divorce, can be associated with negative psychological impacts in the child. Further larger studies are recommended to explore this area further in Sri Lanka. Psychiatrists should also be aware of BSS even when assessing adult patients, since negative experiences in their childhood due to early separation from parents as a result of being boarded may have a long-term impact on adult emotions and behaviours.

\section{Acknowledgments}

The author expresses sincere gratitude to Dr W A L Welgama and Dr G Tilakaratne of the psychiatry unit of Base Hospital Diyatalawa, without whom this work would not have been a possibility.

\section{Declaration of interests}

There are no conflicts of interests

AS Malalagama, Consultant Psychiatrist, Base Hospital Diyatalawa, Sri Lanka

Corresponding author: AS Malalagama

Email: shakunthini@yahoo.com

http://orcid.org/0000-0002-5507-9815

\section{References}

1. Amarasinghe H, Ratnayake P. Role of Education in Economic Development: The Experience of Sri Lanka. SAGA university. Sri Lanka.2008.

Retrieved from http://www2.rikkyo.ac.jp/web/abeyo97/ HPkeisaiyou/2- c.pd

2. Schaverien J. Boarding School Syndrome: Broken attachments a hidden trauma. Br J Psychother 2011; 27: 138-55.

3. Bifulco AT, Brown GW, Harris TO. Childhood loss of parent, lack of adequate parental care and adult depression: a replication. J Affect Disord 1987; 12: 115-28. 
4. Schaverien J. Boarding school: the trauma of the 'privileged' child. J Anal Psychol 2004; 49: 683-705.

5. Bramston P, Patrick J. Rural adolescence experiencing an urban transition. Aust J Rural Health 2007; 152: 247-51.

6. Connell JP, Wellborn JG. Competence, autonomy, and relatedness: a motivational analysis of self-system processes. In: Gunnar MR, Sroufe LA, eds. Self Processes and Development: the Minnesota Symposia on Child Development, 23. Erlbaum, Hillsdale, NJ, 1991: 43-78.

7. Deci E, Vallerand R, Pelletier L, Ryan R. Motivation and education: The self determination perspective. Educ Psychol 1991; 26: 325-46.

8. Earls F, Carlson M. The social ecology of child health and well-being. Annu Rev Publ Health 2001; 22: 143-166.

9. Malalagama AS, Tennakoon S, Abeyasinghe DRRA. Validation of Adolescent Suicide Assessment Protocol-20 (ASAP-20) to Sri Lankan adolescents. Asian J Psychiatr 2018; 33: 11-17.

10. Dorabawila SSKBM, Samita S, Thattil RO. Classification of state schools in Sri Lanka: A multivariate approach. Ceylon Journal of Science: Physical Sciences 2002; 9(1): 102-9.

11. Braun V, Clarke V. Using thematic analysis in psychology, Qual Res Psychol 2006; 3:(2) 77-101.

12. World Health Organization. The ICD-10 classification of mental and behavioural disorders: clinical descriptions and diagnostic guidelines. Geneva,1992.
13. Zhengkui L, Xinying L, Xiaojia G. "Left Too Early: The Effects of Age at Separation From Parents on Chinese Rural Children's Symptoms of Anxiety and Depression”, Am J Public Health 2009; 99(11): 2049-54.

14. Partridge S. Boarding School Syndrome. Br J Psychother. 2012; 28 (3): 138-55.

15. Newcorn JH, Strain J. Adjustment Disorder in Children and Adolescents, J Am Acad Child Psy 1992; 31(2) 1992: 318-26.

16. Smith C, Carlson BE. "Stress, Coping, and Resilience in Children and Youth”, Soc Serv Rev 1997; 71(2): 231-56.

17. Cauley KM, Jovanovich D. Developing an effective transition program for students entering middle school or high school. The Clearing House: A Journal of Educational Strategies, Issues and Ideas 2006; 80(1): 15-25.

18. Scoliers G, Portzky G, Madge N, et al. Reasons for adolescent deliberate self-harm: a cry of pain and/or a cry for help? Findings from the child and adolescent self-harm in Europe (CASE) study. Soc Psychiat Epidemiol 2009; 44: 601.

19. Mullen PE, Martin JL, Anderson JC, Romans SE, Herbison GP. The long-term impact of the physical, emotional, and sexual abuse of children: A community study. Child Abuse Negl 199; 20(1): 7-21.

20. Bottoms G. Improving schools are trying new approaches to raise achievement of struggling students. In Opening doors to the future: Preparing low-achieving middle grades students to succeed in high school, Atlanta: Southern Regional Education Board 2002; 41-56. 